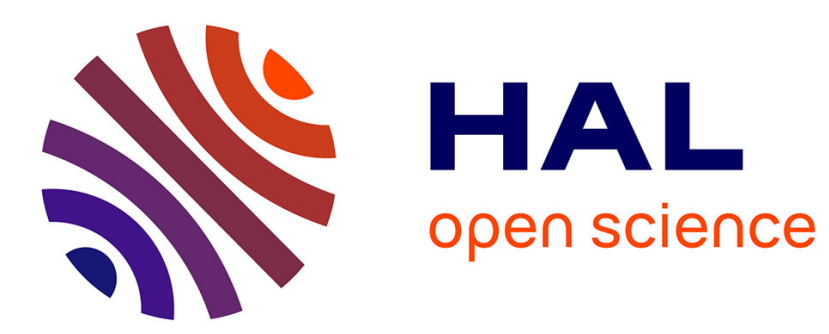

\title{
A reduced model of DA neuronal dynamics that displays quiescence, tonic firing and bursting
}

\author{
Andrew Oster, Boris Gutkin
}

\section{To cite this version:}

Andrew Oster, Boris Gutkin. A reduced model of DA neuronal dynamics that displays quiescence, tonic firing and bursting. Cinquième conférence plénière française de Neurosciences Computationnelles, "Neurocomp'10", Aug 2010, Lyon, France. hal-00553447

\section{HAL Id: hal-00553447 \\ https://hal.science/hal-00553447}

Submitted on 26 Mar 2011

HAL is a multi-disciplinary open access archive for the deposit and dissemination of scientific research documents, whether they are published or not. The documents may come from teaching and research institutions in France or abroad, or from public or private research centers.
L'archive ouverte pluridisciplinaire HAL, est destinée au dépôt et à la diffusion de documents scientifiques de niveau recherche, publiés ou non, émanant des établissements d'enseignement et de recherche français ou étrangers, des laboratoires publics ou privés. 


\title{
A REDUCED MODEL OF DA NEURONAL DYNAMICS THAT DISPLAYS QUIESCENCE, TONIC FIRING AND BURSTING
}

\author{
Andrew M. Oster ${ }^{1,2}$, Boris S. Gutkin ${ }^{1-3}$ \\ andrew.oster@ens.fr, boris.gutkin@ens.fr \\ 1.) Group for Neural Theory \\ 2.) LNC - INSERM Unité 960 \\ Département d'Études Cognitives \\ École Normale Supérieure \\ 29, Rue d'Ulm, 75005, Paris, France \\ 3.) Centre National de la Recherche Scientifique
}

\begin{abstract}
The dopaminergic (DA) neurons in the ventral tegmental area (VTA) project to the amygdyla, prefrontal cortex, and the ventral striatum, and are thought to signal reward prediction error. Numerous studies have focused on the tonic and phasic activity of the VTA DAergic neurons as encoding reward related signals. However, recent studies (e.g., [1]) suggest that bursting behavior specifically conveys reward-related information. Moreover, dopamine release during burst firing events is substantially greater than during regular spiking [2]. In addition, DAergic neuronal bursting is influenced by the nicotinic acetycholine receptors (nAChRs) [3, 4]. Thus, understanding burst firing is essential when considering VTA neuronal dynamics. Here we introduce a single compartmental model of a VTA DA neuron. We demonstrate that this model captures the essential qualitative behavior of DAergic neuronal dynamics, yet is simple compared to existing, detailed, multi-compartmental models. Moreover, this model could be extended to include the detailed dynamics of two prevalent nAChR subtypes ( $\alpha 4 \beta 2$ and $\alpha 7$ ), which is critical to elucidate the role that these receptors play in nicotine addiction.
\end{abstract}

\section{KEY WORDS}

dopamine, bursting, modeling, addiction

\section{Introduction}

The neurotransmitter dopamine (DA) has been implicated in many neural and cognitive processes: motivation, sleep, mood, attention, working memory, learning, punishment and reward. DAergic neurons are predominantly located within the ventral tegmental area (VTA) and the substantia nigra pars compacta of the midbrain. Dopaminergic neurons send numerous projections to cortical and sub-cortical areas and diffusely release dopamine to their targets when the DA neuron fires.

DAergic neurons display a wealth of dynamic behavior: pacemaker-like firing (seen in slice preparations), pe- riods of burst activity, quiescence, and also sporadic activity in vivo (see e.g. [5, 6, 7]). Given this rich behavior and due to their biological importance, much work has been done on modeling DAergic neurons. Certain existing models only consider the subthreshold behavior of the DA cells $[8,9]$ and argue by analogy the expected behavior of the DA cells. Other models depend on the interaction between the activity at the soma and the dendrites $[10,11,9]$ in order to produce bursting dynamics (with this approach certain parameters may be unobservable). However, recent experimental findings [12, 13] indicate that burst firing can be generated somatically with the dendrites silenced. These somatically induced bursts had characteristics consistent with normal bursting, suggesting that a single-compartmental model should be sufficient for generating the observed DA neuronal dynamics. In this work, we introduce such a model for DA neuronal dynamics.

\section{Biological Background}

The DA cells within the VTA are thought to transmit reward prediction error [14]. Such a reward prediction error signal would be of critical importance in the the actionselection process that ultimately fashions animal behavior.

The acute affect of nicotine is associated with an immediate increase in DA outflow. Studies of the reinforcing effects of self-administered intravenous nicotine on rodents have focused on the activation of nicotinic acetylcholine receptors (nAChRs) within the mesocorticocolimbic dopaminergic system, which projects from the VTA to the nucleus accumbens (NA) and the prefontal cortex (PFC) $[15,16,17,18]$. Anatomical studies suggest that VTA DA cells have high levels of nAChRs [19]. Nicotine affects the dopaminergic system through binding to the nAChR complexes [20], which activates then desensitizes these receptors. The nAChRs are present both on the DAergic cells as well as GABAergic neurons within the VTA. The expression level of the nAChRs, that is, their density and subreceptor compositions, likely differs between the DAergic and GABAergic populations. Nicotine activation 
of the nAChRs leads to increased averaged activity of the DAergic cells, that is, exposure to nicotine induces a phasic response in the DA cell activity. The pathway for this phasic response, may be through direct excitation of the DAergic neurons or via nicotine affecting the GABA cells causing a disinhibition of the DA neuronal population.

The phasic response of the DA cells is not only an increase in the irregular pacemaker firing, but is also associated with an increase in burst firing [4] (bursts being typically 2-6 spikes in quick succession). Recent work by Graupner and Gutkin [21] suggested a simplified neural architecture for the VTA network, seen below in Figure 1, can account for the phasic increased response of the VTA circuit using an averaged activity model. We will adopt a similar network architecture as a backdrop to our construction of a DA neuronal model; however, in this work we will not be directly considering the effects nicotine on DA cell behavior.

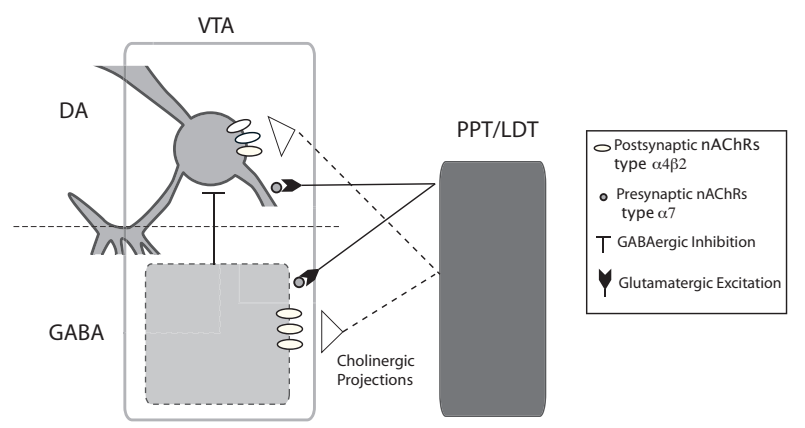

Figure 1. Simplified model of the DA/GABA VTA circuit. The neural drive to the system from the pedunculopontine and laterodorsal tegmental areas (PPT/LDT) innervates both the DA and GABA neuronal populations.

\section{DA Cell Model}

We introduce a single compartmental conductance based model for the dynamics of a DA neuron that combines conductance mechanisms previously included in $[11,8]$. The dynamics of the membrane potential are taken to be

$$
\begin{aligned}
C_{m} \frac{d V}{d t}= & I_{\text {applied }}-I_{C a}^{L}(V)-\chi_{A P A} I_{K}^{S K}(V, u) \\
& -I_{K}(V)-I_{\text {leak }}-I_{G A B A} \\
& -\chi_{T T X}\left[I_{N a}(V, h)+I_{K}^{d r}(V, n)\right. \\
& \left.+I_{N M D A}(V)\right]
\end{aligned}
$$

where $I_{\text {applied }}$ is a generic input to the cell that represents an applied current in vitro or the tonic input to the area in vivo. The GABA neuronal population inhibits the DA neuronal population. The drive to both the GABA and DA circuits is a combination of cholinergic and glutamatergic inputs and are sensitive to nicotine. In this work, we will not explicitly track the dynamics for the GABA circuit, but lump the inhibitive effects into the averaged applied current, that is, we take $I_{G A B A}=0$. We include a general leak current, $I_{\text {leak }}=0.02\left(E_{\text {leak }}-V\right)$ with $E_{\text {leak }}=-50 \mathrm{mV}$. Additionally, the potassium current is given by

$$
I_{K}(V)=\bar{g}_{K}\left(\frac{1}{1+\exp \frac{-\left(V-k_{1}\right)}{k_{2}}}\right)\left(E_{K}-V\right) .
$$

with $E_{K}=-90 \mathrm{mV}$ (other parameters listed in appendix).

The L-type calcium current, $I_{C a}^{L}$, is counterbalanced with the apamine sensitive SK-type potassium current, $I_{K}^{S K}$. The SK current is dependent on both the voltage and calcium level, with calcium concentration denoted by $u$.

The L-type calcium current is of the form

$$
I_{C a}^{L}(V)=G_{C a}^{L}(V)\left(E_{C a}-V\right)
$$

with $G_{C a}^{L}$ given in the appendix and $E_{C a}=100 \mathrm{mV}$.

Whereas the SK potassium current is given by

$$
I_{K}^{S K}(V, u)=G_{S K}(u)\left(E_{K}-V\right)
$$

where $G_{S K}(u)=\bar{g}_{S K}\left(\frac{u^{4}}{u^{4}+K_{1}^{4}}\right)$.

Calcium enters the cell predominantly via the L-type calcium channel (with a minor contribution due to the NMDA channel). The $\mathrm{Ca}^{2+}$ is then ejected via a pump. Thus, calcium, $u$, varies as

$$
\frac{d u}{d t}=\frac{2 f_{C a}}{r}\left(\frac{I_{C a}^{L}+I_{N M D A}^{C a}}{H}-I_{p u m p}\right)
$$

where the cytosolic buffering constant is given by $f_{C a}=$ $0.01, H$ is a lumped term involving the valence of calcium and Faraday's number, the radius, $r$, of the neuron is taken to be $r=20 \mu \mathrm{m}$, and the pump dynamics are taken to be (akin to [9]):

$$
I_{\text {pump }}(u)=\frac{M_{\text {pump }} u}{\left(u+K_{\text {pump }}\right)}
$$

with $M_{\text {pump }}=350$ and $K_{\text {pump }}=700$.

\subsection{Subthreshold Behavior: Modeling Phar- macology}

Tetrodotoxin (TTX) blocks the sodium current. Without the sodium dynamics, no spikes occur and several other processes requiring spikes(referred to as active processes) are rendered redundant; the delayed rectifying current $\left(I_{K}^{d r}\right)$ and NMDA $\left(I_{N M D A}\right)$ current are both taken to be silenced. Under TTX application $\left(\chi_{T T X}=0\right)$, our model captures the qualitative subthreshold activity. As seen in Figure $2 a$, the system exhibits a slowly oscillating potential (SOP) [22] that is hypothesized to play a role in providing the underlying structure for regular firing (e.g., [8]).

With the further addition of apamine, the SK-channels are silenced and the L-type calcium signal dominates the 
subthreshold behavior [23]. The parameter $\chi_{A P A}$ is associated with the strength of the SK channels, setting $\chi_{A P A}=$ 1 signifies no apamine applied whereas $\chi_{A P A}=0$ would signify the complete blockage of SK channels due to a high concentration of apamine. In Figure $2 b$, the produced voltage trace exhibits a square-wave-like behavior with long plateaus of depolarization (similar to experimental findings, e.g., [23]). It is hypothesized that upon these plateaus of depolarization burst firing is initiated, suggesting that burst generation critically depends upon the calcium dynamics [5]. a)

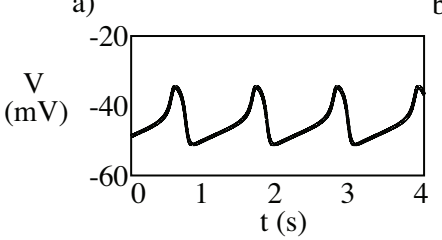

b)

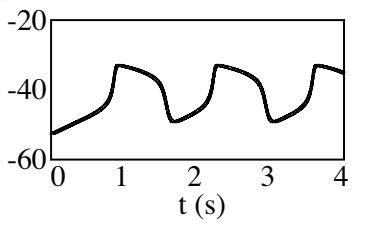

Figure 2. The subthreshold behavior of a DA neuron. In (a), note the slowly oscillating potential (SOP), whereas, in (b) there are plateaus of depolarization where burst events are hypothesized to occur. The values for $\chi_{A P A}$ are 1 and 0.025 in (a) and (b), respectively.

\subsection{Active and Synaptic Processes}

Earlier works examine only the subthreshold, silenced behavior and then by analogy suggest what the active behavior should be (e.g., [8]). We explicitly model the fast processes associated with spike generation; these depend critically on sodium. The sodium dynamics are taken to be Morris-Lecar-like with fast activation and slower inactivation, that is,

$$
I_{N a}=\bar{g}_{N a} m_{\infty}^{3}(V) h\left[V-E_{N a}\right]
$$

with the conductance $\bar{g}_{N a}=150$ and the reversal potential given as $E_{N a}=55 \mathrm{mV}$. The fast activation is taken to be of the form

$$
m_{\infty}(V)=\frac{1}{2}\left[1-\tanh \left(\frac{p_{2}-V}{p_{3}}\right)\right]
$$

with $p_{2}=-11 \mathrm{mV}$ and $p_{3}=19$. The slower inactivation evolves according to

$$
\frac{d h}{d t}=\alpha_{h}(v)(1-h)-\beta_{h}(v)
$$

where $\alpha_{h}(V)=\frac{h_{a 1}}{2}\left[1+\tanh \left(\frac{h_{a 2}-V}{h_{a 3}}\right)\right]$ and $\beta_{h}(V)=\frac{h_{b 1}}{2}\left[1-\tanh \left(\frac{h_{b 2}-V}{h_{b 3}}\right)\right]$.

In addition, we include a delayed-rectifier potassium current of the form

$$
I_{K}^{d r}(V)=\bar{g}_{K} n^{4}(V)\left[V-E_{K}\right]
$$

where the conductance is given by $\bar{g}_{K}=4$ and the reversal potential $E_{K}=-90 \mathrm{mV}$. The associated gating variable evolves according to

$$
\frac{d n}{d t}=\alpha_{n}(V)(1-n)-\beta_{n}(V) n
$$

with $\alpha_{n}(V)=\frac{n_{a 1}}{2}\left[1-\tanh \left(\frac{n_{a 2}-V}{n_{a 3}}\right)\right]$ and $\beta_{n}(V)=\frac{n_{b 1}}{2}\left[1+\tanh \left(\frac{n_{b 2}-V}{n_{b 3}}\right)\right]$.

Beyond fast sodium and potassium, the NMDA currents are also of import

$$
I_{N M D A}=G_{N M D A}(V)\left[V-E_{N M D A}\right]
$$

and

$$
G_{N M D A}(V)=\left(\frac{\bar{g}_{N M D A}^{s t i m}+\bar{g}_{N M D A}^{c}}{1+0.28(M g) e^{-m e *(V+20)}}\right)
$$

where $M g$ denotes the amount of magnesium, $0.5 \mu \mathrm{M}$. Here $E_{N M D A}=0, \bar{g}_{N M D A}^{\text {stim }}=0.1, \bar{g}_{N M D A}^{c}=0.01$, and $m e=$ 0.08 . The parameter $\bar{g}_{N M D A}^{\text {stim }}$ is zero, except during periods corresponding with NMDA activation.

\subsection{Quiescence and Tonic Behavior}

For sufficiently small $I_{\text {applied }}$, the model can display quiescence (not shown). As $I_{\text {applied }}$ increases, the system can undergo a change in behavior and firing would occur (The critical value of $I_{\text {applied }}$ for the bifurcation will depend upon the parameters chosen). The dynamics produced depend critically on the parameter $\chi_{a p a}$, which represents the relative strength of the SK-current (and its value can also be associated with the administration of apamine). Below in Figure 3, we demonstrate that the DA neuron can display slow pacemaker-like tonic firing given sufficient input.

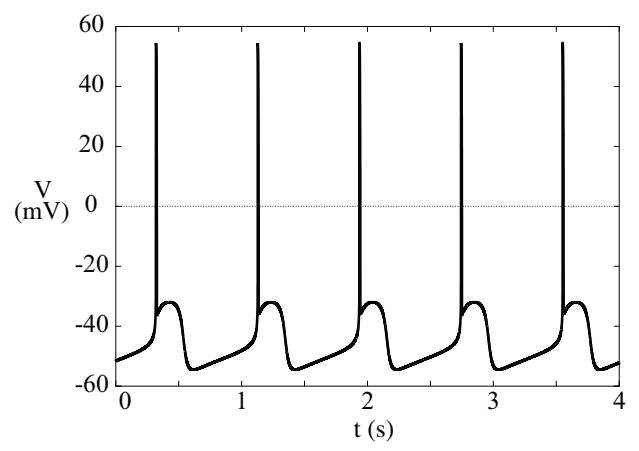

Figure 3. The DA neuron can display tonic firing with an background input of $I_{\text {applied }}=0.4$. No apamine is applied, so $\chi_{A P A}=1$. Here the activation of Iapplied $=0.4$. No apamine is applied, so $\chi_{A P A}=1$. Here the activation of
the sodium current variable $p_{2}$ is slightly modified to $-8 \mathrm{mV}$, whereas otherwise it is $-11 \mathrm{mV}$. 


\subsection{Bursting Dynamics}

\subsubsection{Defining bursting}

The seminal work of Grace and Bunney [5] examined the properties of the in vivo bursts and introduced criteria for identifying the bursts. Burst onset was associated with a tight doublet of two successive spikes. In particular, burst onset was defined as "the concurrence of two spikes with an interspike interval (ISI) of less than $80 \mathrm{~ms}$. Burst termination was defined as an interspike interval of over $160 \mathrm{~ms}$." This is a straightforward, algorithmic definition of a burst and still commonly practiced today. With this definition, one often employs a measure of the degree of burst firing, that is, percentage of spikes occurring within bursts. This measure of bursting behavior breaks down with increased activity as it treats fast, tonic firing to be equivalent to a burst of extremely long duration. This can be contrasted with a recently developed measure for degree of bursting [24]:

$$
B=\frac{2 \operatorname{var}\left(t_{i+1}-t_{i}\right)-\operatorname{var}\left(t_{i+2}-t_{i}\right)}{2 E^{2}\left(t_{i+1}-t_{i}\right)}
$$

where $t_{i}$ denotes the time of the $i$ th spike, $E$ and var denote the expected value and variance of the spike times, respectively. One virtue of this measure, is that it varies continuously with time so one has an online representation of the degree of bursting within a spike train. Using this measure, $B$ above a threshold value, $B_{\Theta}$, would constitute bursting. For example, in [24], a neuron with a measure $B>B_{\Theta}=0.15$ was considered to be bursting.

\subsubsection{Endogenous bursting}

Experimentally Ping and Sheperd [23] have observed that application of apamine induces burst firing by acting upon the SK-channels, reducing the flow of $\mathrm{K}^{+}$. Here we simulate the application of apamine by reducing the parameter $\chi_{A P A}$. Under these conditions, the model exhibits endogenous or "natural" bursting.

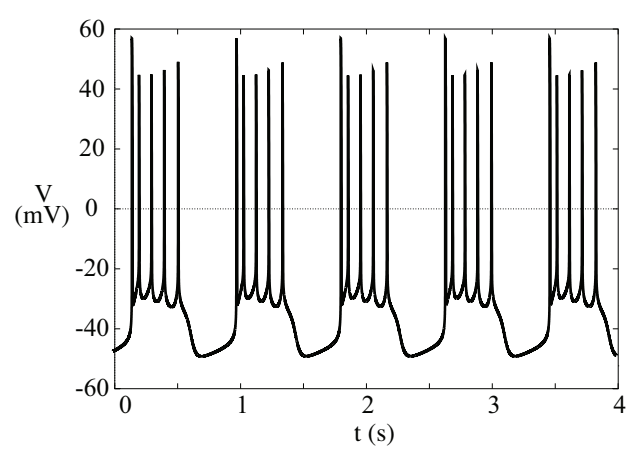

Figure 4. A DA neuron exhibiting burst firing with an background input of $I_{\text {applied }}=0.4$ and with an application of apamine, so that $\chi_{A P A}=0.025$.
The bursts shown in Figure 4 have the property that within a burst, the second spike shortly follows the initial spike, that is, the bursting displays the characteristic initial tight spike doublet at burst onset. The burst measure given in Equation 13 is $B_{0.4} \approx 0.23$. Here $I_{\text {applied }}=0.4$. For slightly larger applied current $I_{\text {applied }}=0.5$, the average activity of the neuron increases and the interburst duration decreases, with the number of spikes per burst decreasing (simulation not shown). Using the burst measure (Equation 13), this would signify a decrease in amount of bursting (or "burstiness"). For even larger $I_{\text {applied }}$ (e.g., $I_{\text {applied }}=$ 1.5), the DAergic neuron displays fast tonic firing, shown in Figure 5. This suggests a phase diagram can be produced (a cartoon of which is pictured in Figure 6) outlining the different dynamical behaviors during apamine application as one varies the applied current.

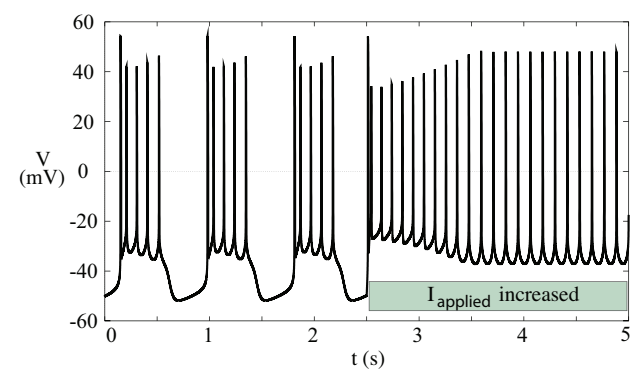

Figure 5. A transition from bursting to tonic behavior occurs with increased applied current (apamine is applied in this preparation). Here $\chi_{A P A}=0.025$ with $I_{\text {applied }}=0.4$ initially and then increases at $t=2.5$ s to $I_{\text {applied }}=1.5$.

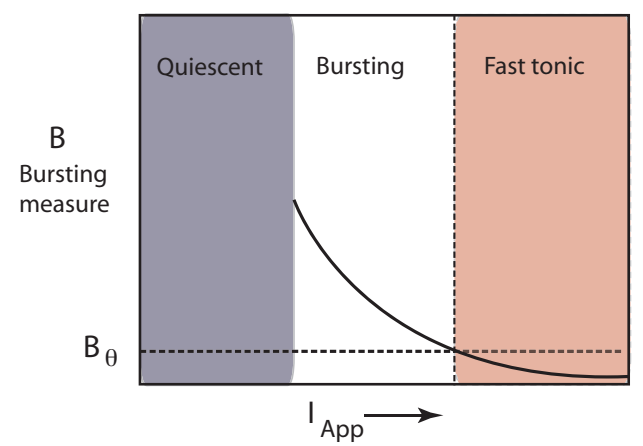

Figure 6. Schematic of the transitions from quiescence to bursting to fast tonic behavior as $I_{\text {applied }}$ increases in the case of a weak SK-current $(\chi A P A=$ 0.025). Here the bursting measure is shown on the vertical axis.

\subsubsection{Stimulus induced bursting}

Thus far, we have examined the emergence of an endogenous bursting behavior by altering the strength of the SK-potassium channel through application of the drug apamine. However, there is another pathway to obtain bursting, that is, stimulus induced bursting. A transient increase in NMDA conductance results in an increase in the firing rate. This bursting event does not display 
burst/interburst periods, but rather an increase in activity for the duration of the stimulus, as shown in Figure 7 , where at time $2.2 \mathrm{~s} g_{N M D A}$ is increased to 0.1 for a duration of $1.5 \mathrm{~s}$.

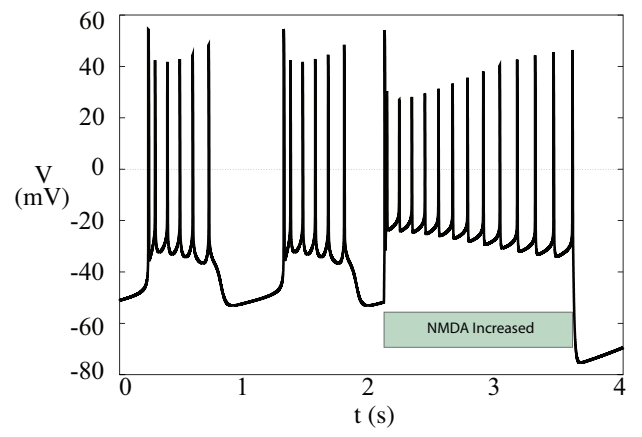

Figure 7. A DA neuron exhibiting burst firing with an background input of $I_{\text {applied }}=0.3$ and $\chi_{A P A}=0.025$. At time $t=2.2 \mathrm{~s}, \mathrm{NMDA}$ is applied (manifested by the NMDA conductance immediately being raised to a value of 0.1 ), which elicits a sustained bursting event for the duration of the NMDA application. Note that although the neuron is initially bursting, it is not a necessary condition for stimulus-induced bursting.

\section{Conclusions}

As burst firing is associated with high levels of dopamine release (compared to regular spikes train), the understanding of the mechanisms by which DA neurons enter (or fall out of) burst firing regimes may play a role in pinpointing therapies for disorders involving dopamine (e.g., Parkinsons, Alzheimers, and addiction). Our model captures many of the observable patterns of DAergic neuronal activity: quiescence, slow pacemaker-like tonic firing, endogenous bursts, fast regular spiking, and an NMDA-dependent stimulus-induced "burst" firing. The "burst" firing due to an increase in NMDA would necessarily strongly correlate with concerted synaptic drive necessary to create the elevated levels of NMDA.

The necessary conditions to induce endogenous bursting are a suppression of the SK potassium current and a sufficiently strong input tone to the DA neuron. SK channel decreases are of potential physiological significance since such slow K-channels are the primary target for downmodulation by Ach through muscarinic AChRs (mAChRs), hence Ach action through mAChRs may prime the DA neurons for bursting (e.g., [25]), while the increases in the drive, either synaptic or through nicotinic mechanisms would then result in burst. The strength of this drive would depend upon the level of excitation compared to the amount of inhibition received from the GABAergic neuronal population. Additionally, some DA neurons may intrinsically have a lower density of SK channels; recent work has uncovered at least two distinct DA neural populations interspersed within the VTA[26]. Our model provides a framework for future study to determine the dominant pathway to burst firing. Our most immediate future direction is to include the $\mathrm{nAChR}$ dynamics in order to examine the effects of nicotine on burst firing. The model results and predictions are to be contrasted with the past and recent findings by Mameli-Engvall et al. [4].

\section{Acknowledgements}

The authors would like to thank Philippe Faure (Univ. Paris VI) and Mark Humphries (ENS) for many helpful discussions. Support for this work was provided by: ANR MNP "Dopanic" (BG, AO), NeRF postdoctoral fellowship (AO), CNRS (BG), INSERM (BG), and an ENP collaborative grant $(B G)$.

\section{Appendix}

The L-type $\mathrm{Ca}^{2+}$ terms:

$$
G_{C a}^{L}(V)=\bar{g}_{C a}^{L}\left(\frac{\alpha_{C}(V)}{\alpha_{C}(V)+\beta_{C}(V)}\right)^{4}
$$

with

$$
\alpha_{C}(V)=-0.0032 \frac{(V+50)}{\exp (-(V+50) / 5)-1}
$$

and

$$
\beta_{C}(V)=\exp \left(\frac{-(V+55)}{40}\right)
$$

Simulation Parameters

\begin{tabular}{|c||c|c|c|}
\hline variable & & & \\
\hline \hline$\alpha_{h}$ & $h_{a 1}=0.01$ & $h_{a 2}=-60$ & $h_{a 3}=18$ \\
\hline$\beta_{h}$ & $h_{b 1}=0.5$ & $h_{b 2}=10$ & $h_{b 3}=5$ \\
\hline$\alpha_{n}$ & $n_{a 1}=1$ & $n_{a 2}=100$ & $n_{a 3}=70$ \\
\hline$\beta_{n}$ & $n_{b 1}=2$ & $n_{b 2}=-30$ & $n_{b 3}=15$ \\
\hline$I_{S K}$ & $\bar{g}_{S K}=8$ & $K_{1}=1$ & \\
\hline$I_{K}$ & $\bar{g}_{K}=0.4$ & $k_{1}=-15$ & $k_{2}=7$ \\
\hline$I_{C a}^{L}$ & $\bar{g}_{C a}^{L}=8$ & & \\
\hline
\end{tabular}

\section{References}

[1] W. Schultz. Getting formal with dopamine and reward. Neuron, 36(2):241-263, October 2002.

[2] F. G. Gonon. Nonlinear relationship between impulse flow and dopamine released by rat midbrain dopaminergic neurons as studied by in vivo electrochemistry. Neuroscience, 24(1):19-28, Jan 1988.

[3] J. Grenhoff, G. Aston-Jones, and T.H. Svensson. Nicotinic effects on the firing pattern of midbrain dopamine neurons. Acta Physiol Scand, 128(3):3518, 1986. 
[4] M. Mameli-Engvall, A. Evrard, S. Pons, U. Maskos, T. H. Svensson, J-P Changeux, and P. Faure. Hierarchical control of dopamine neuron-firing patterns by nicotinic receptors. Neuron, 50(6):911-921, Jun 2006.

[5] A. A. Grace and B. S. Bunney. The control of firing pattern in nigral dopamine neurons: burst firing. $J$ Neurosci, 4(11):2877-2890, Nov 1984.

[6] A. A. Grace and B. S. Bunney. The control of firing pattern in nigral dopamine neurons: single spike firing. J Neurosci, 4(11):2866-2876, Nov 1984.

[7] P. G. Overton and D. Clark. Burst firing in midbrain dopaminergic neurons. Brain Res Brain Res Rev, 25(3):312-334, Dec 1997.

[8] B. Amini, J. W. Clark, and C. C. Canavier. Calcium dynamics underlying pacemaker-like and burst firing oscillations in midbrain dopaminergic neurons: a computational study. J Neurophysiol, 82(5):22492261, Nov 1999.

[9] C. C. Canavier, S. A. Oprisan, J. C. Callaway, H. Ji, and P. D. Shepard. Computational model predicts a role for ERG current in repolarizing plateau potentials in dopamine neurons: Implications for the modulation of neural activity. J Neurophysiol, 98:30063022, 2007.

[10] C. J. Wilson and J. C. Callaway. A coupled oscillator model of the dopaminergic neuron of the substantia nigra. J. Neurophysiol., 83:3084-3100, 2000.

[11] A. S. Kuznetsov, N. J. Kopell, and C. J. Wilson. Transient high-frequency firing in a coupled-oscillator model of the mesencephalic dopaminergic neuron. $J$ Neurophysiol, 95(2):932-947, Feb 2006.

[12] S. N. Blythe, D. Wokosin, J. F. Atherton, and M. D. Bevan. Cellular mechanisms underlying burst firing in substantia nigra dopamine neurons. J. Neurosci., 29(49):15531-15541, 2009.

[13] C. A. Deister, M. A. Teagarden, C. J. Wilson, and C. A. Paladini. An intrinsic neuronal oscillator underlies dopaminergic neuron bursting. J. Neurosci., 29(50):15888-15897, 2009.

[14] W. Schultz. Predictive reward signal of dopamine neurons. J Neurophysiol, 80(1):1-27, Jul 1998.

[15] W. A. Corrigall, K. Franklin, K. Coen, and P. Clarke. The mesolimbic dopaminergic system is implicated in the reinforcing effects of nicotine. Psychopharmacology, 107(2):285-289, June 1992.

[16] W. A. Corrigall, K. M. Coen, and K. L. Adamson. Self-administered nicotine activates the mesolimbic dopamine system through the ventral tegmental area. Brain Res, 653(1-2):278-284, Aug 1994.
[17] M. Nisell, G. G. Nomikos, and T. H. Svensson. Systemic nicotine-induced dopamine release in the rat nucleus accumbens is regulated by nicotinic receptors in the ventral tegmental area. Synapse, 16(1):36-44, Jan 1994.

[18] F. E. Pontieri, G. Tanda, F. Orzi, and G. Di Chiara. Effects of nicotine on the nucleus accumbens and similarity to those of addictive drugs. Nature, 382(6588):255-257, July 1996.

[19] P. B. S. Clarke and A. Pert. Autoradiographic evidence for nicotine receptors on nigrostriatal and mesolimbic dopaminergic neurons. Brain Res., 348:355-358, 1985.

[20] J. P. Changeux, D. Bertrand, P. J. Corringer, S. Dehaene, S. Edelstein, C. Léna, N. Le Novére, L. Marubio, M. Picciotto, and M. Zoli. Brain nicotinic receptors: structure and regulation, role in learning and reinforcement. Brain Res Brain Res Rev, 26(2-3):198216, May 1998.

[21] M. Graupner and B. Gutkin. Modeling nicotinic neuromodulation from global functional and network levels to nAChR based mechanisms. Acta Pharmacol Sin, 30(6):681-686, 2009.

[22] Y. Kang and S.T. Kitai. A whole cell patch-clamp study on the pacemaker potential in dopaminergic neurons of rat substantia nigra compacta. Neuroscience Research, 18(3):209 - 221, 1993.

[23] H. X. Ping and P. D. Shepard. Apamin-sensitive $\mathrm{Ca}(2+)$-activated $\mathrm{K}+$ channels regulate pacemaker activity in nigral dopamine neurons. Neuroreport, 7(3):809-14, 1996.

[24] R. A. J. van Elburg and A. van Ooyen. A new measure for bursting. Neurocomputing, 58-60:497-502, 2004.

[25] G. Drion, M. Bonjean, O. Waroux, J. Scuvée-Moreau, J-F Liégeois, T. J. Sejnowski, R. Sepulchre, and V. Seutin. M-type channels selectively control bursting in rat dopaminergic neurons. Eur J Neurosci, $31: 827-835,2010$.

[26] S. Lammel, A. Hetzel, O. Häckel, I. Jones, B. Liss, and J. Roeper. Unique properties of mesoprefrontal neurons within a dual mesocorticolimbic dopamine system. Neuron, 57:760-773, 2008. 